\title{
Research on Teacher Evaluation System of Private Universities Based on Support Vector Machine Yang Bo ${ }^{1, a}$, Zhang Li-na ${ }^{2, b}$ \\ ${ }^{1}$ Changchun University of Finance and Economics, Changchun, Jilin, 130122, China \\ 2 Jilin Agricultural University, Changchun, Jilin, 130118, China \\ amail_yangbo@163.com, ${ }^{\mathrm{a}}$ mail_zhangln@163.com
}

Keywords: teacher evaluation, support vector machine, index of teacher evaluation, intelligent evaluation

\begin{abstract}
This study applies the support vector machine theory to the private university teacher evaluation system. According to the theory of educational evaluation, combined with the current evaluation indexes of teachers in private universities, this study makes a scientific and reasonable setting for the evaluation indexes of private universities. Based on the principle of support vector machine (SVM), the sample data is divided into two parts, by training the sample data in the evaluation system, the training model is obtained, and the prediction data are evaluated and analyzed with the training model. Through the experiment and trial operation, it is indicated that the evaluation method based on the support vector machine can make the evaluation more accurate and reasonable, and it can reduce some errors that caused by human factors in the process of evaluation.
\end{abstract}

\section{Introduction}

The teaching evaluation method based on computer intelligence is to study the problem of teacher evaluation in the field of artificial intelligence, such as related disciplines (Such as neural network, fuzzy logic, etc.) and data mining methods, and achievements in scientific research in this field. Namely, to seek a kind of teacher evaluation method based on the experience and knowledge of experts in the field and computer technology. By this method, we not only can evaluate teachers' teaching quality and teaching level and comprehensive ability, but also can evaluate some other phenomena in the field of education.

\section{Introduce support vector machine}

Support vector machine based on statistical learning theory and structural risk minimization principle, SVM Have outstanding advantages in solving practical problems, such as small samples, nonlinear, local minimum points and high dimensions. It has been successfully applied in pattern classification, time series prediction and function approximation and so on. The problem of teaching evaluation can be regarded as a kind of complex classification problem between the teaching ability and the related data set. The main purpose of this study is to introduce the support vector machine theory into the teaching evaluation system of private universities. In the process of teacher evaluation, teachers' evaluation indexes are determined according to the actual situation of the private universities. Then collected evaluate data according to the indexes, the data include the value of each index. We carry out evaluation work based on these sample data sets. From the collected data can be seen if every teacher evaluation data includes n-index can regard it as an $\mathrm{n}$-dimensional vector, can be expressed as:

$$
\mathrm{X}=\left(\mathrm{X}_{1}, \mathrm{X}_{2}, \mathrm{X}_{3} \ldots \mathrm{X}_{\mathrm{n}}\right)
$$

So for the entire teacher evaluation of the sample data can be expressed as:

$$
\mathrm{Xi}=\left(\mathrm{Xi}_{1}, \mathrm{Xi}_{2}, \mathrm{Xi}_{3} \ldots \mathrm{Xi} \mathrm{i}_{\mathrm{n}}\right) \quad \mathrm{i}=1,2,3 \ldots \mathrm{m}
$$

$\mathrm{N}$ is the number of evaluation indexes in the whole evaluation system, $\mathrm{m}$ is the number of teachers, $\mathrm{X}_{\mathrm{i}, \mathrm{j}}$ is the jth index value of the ith teacher evaluation data. 


\section{Construction principles of teachers' evaluation index}

Teacher evaluation work is complex, because it is affected by many factors, in the establishment of the index system to consider many aspects. To sum up, the following principles should be followed: the principle of science, the principle of fairness, the principle of incentive, the principle of comprehensiveness, the principle of rationality, the principle of development.

The evaluation index system of this study is composed of three dimensions:

$>$ Index I: For the evaluation of teachers will have to study aspects, namely a big classification, every I class contains a number of sub index.

$>$ Index II: Index I refinement of several sub index.

$>$ Index III: Content and standard of evaluation.

According to the characteristics of private university teachers in China, the evaluation index system is established, due to space, some representative indexes are described, as shown in the table 1 .

Table1. Index of teacher evaluation

\begin{tabular}{|c|c|c|}
\hline Index I & Index II & Index III \\
\hline \multirow{3}{*}{ Teaching } & Teaching workload & The number of hours required by teachers to complete \\
\hline & Teaching quality & $\begin{array}{l}\text { Good teaching effect, cultivate the ability to solve practical } \\
\text { problems with the application of knowledge }\end{array}$ \\
\hline & $\begin{array}{c}\text { Teaching } \\
\text { achievement }\end{array}$ & $\begin{array}{l}\text { Novelty, practicality, to improve teaching level and education } \\
\text { quality }\end{array}$ \\
\hline \multirow{3}{*}{$\begin{array}{l}\text { Scie } \\
\text { ntific } \\
\text { research }\end{array}$} & Research project & The number and level of the project to host or participate. \\
\hline & $\begin{array}{l}\text { Published papers } \\
\text { and books }\end{array}$ & $\begin{array}{c}\text { The number of papers published, the number of books and } \\
\text { papers }\end{array}$ \\
\hline & $\begin{array}{l}\text { Scientific research } \\
\text { achievements }\end{array}$ & Get different levels of research awards \\
\hline
\end{tabular}

\section{Design of teacher evaluation system}

This study uses C\# as programming language, uses the LIBSVM-mat toolbox of Matlab to realize the model creation, and finally realizes the private university teacher evaluation system. The system mainly consists of two parts. The first part is the model training of the system; the second part is the prediction of the treatment of the sample. The main task of the model training is to train the private college teacher evaluation system with the training sample data, and then to establish the classification model. The main task of the prediction part is to use the model to evaluate the test samples. The system includes basic information management, system management, and training evaluation management. The basic information management mainly includes the teacher evaluation data acquisition and evaluation index system input. System management includes user management, database management. Training evaluation management mainly includes the establishment of the training model, the treatment of the test data evaluation, evaluation of the results of the query and analysis.

There may be some errors in the process of data acquisition. Therefore, before using the data need to preprocess the sample data set. The pretreatment includes teacher's evaluation data into vector form, and the data were normalized. After normalizing the data, the convergence of training can be improved. After the experiment, we found that the sample data for the evaluation of the teacher will be normalized to the $[0,1]$, the forecast effect is better. Table 2 is comparison of the normalized data and the original data. 
Table2. Comparison of the normalized data and the original data

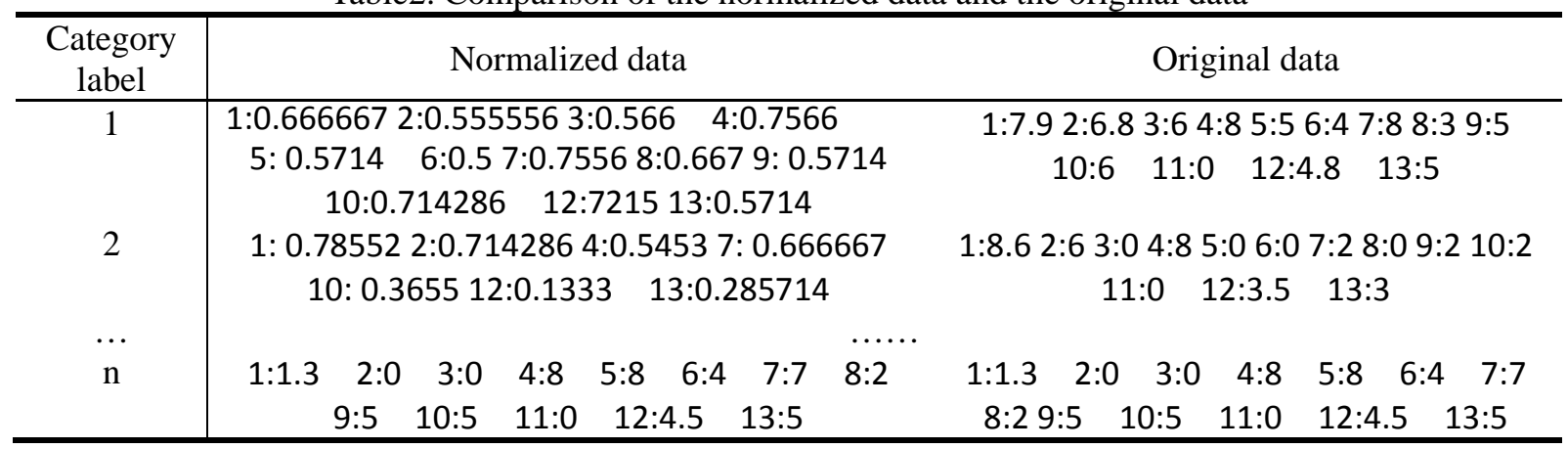

It is very important to choose the proper parameters of the support vector machine. This mainly includes the selection of the type of support vector machine model, they are corresponding to the kernel function, as well as some other parameters. In this study, we select the classic support vector machineC-SVM, C as the punishment factor. Because the punishment factor has a great influence on the final classification result, the greater the value of the value, the greater the punishment on the error classification. In this study, we select different kernel function to carry out the experiment. At first, the RBF kernel function is selected, and the punishment factor is tested. The method is using the same $\gamma$ value. The value of $\gamma$ is the reciprocal of the vector $n$. Using Libsvm-mat to change the value of the punishment parameter of the kernel function, and to see the results. The results of the experimental data are shown in Table 3.

Table3. RBF kernel function is punishment parameter $\mathrm{C}$ experiment

\begin{tabular}{ccc}
\hline Punishment parameter $(C)$ & Kernel function parameter $(\gamma)$ & Accuracy rate \\
\hline$C=2^{1}$ & $v=0.074$ & $63.33 \%$ \\
$C=2^{3}$ & $\gamma=0.074$ & $75.95 \%$ \\
$C=2^{5}$ & $\gamma=0.074$ & $88,21 \%$ \\
$C=2^{6}$ & $\gamma=0.074$ & $91.11 \%$ \\
$C=2^{7}$ & $\gamma=0.074$ & $93.26 \%$ \\
$C=2^{8}$ & $\gamma=0.074$ & $92,76 \%$ \\
\hline
\end{tabular}

We use the same method to experiment with polynomial kernel function and Sigmoid function respectively. The experiment data shows that in the training sample data, the range of punishment factor in $2^{0}-2^{7}$. We find that the improvement of the number of polynomials, the accuracy of the prediction will not improve but will be decreased. When the punishment factor is increased, the corresponding prediction accuracy of the three kinds of kernel functions will increase with the increase of the punishment factor, but when the punishment factor reaches a certain value, the corresponding accuracy will not increase with the increase of the value. By comparison, we choose the value of $2^{7}$ as the punishment factor. A large number of experimental data indicate that the RBF function is relatively stable in terms of prediction accuracy. Therefore, the RBF function is chosen as the kernel function. Another key parameter affecting the accuracy is $\gamma$. We use the same punishment factor to carry out the experiment of the parameter $\gamma$ with the sample data. From the experimental data, we can see that with the increase of $\gamma$ value accuracy rate is rising, but the rate of accuracy is not very large. The prediction accuracy is better when the value of $\gamma$ is in the range of 0.01-0.09. Using RBF as a kernel function, the accuracy rate is $93.26 \%$ when the experimental setting parameters $C=2^{7}$ and $\gamma=0.074$. This study using cross validation method, through a large number of experimental data to choose the appropriate kernel function and the corresponding parameters, obtained through the contrast test of the punishment factor and parameter value. Using the model to validate the prediction samples, the accuracy of the model is satisfied.

\section{Summary}

In this study, support vector machine theory is introduced into the evaluation of private university teachers, according to the characteristics of Chinese private universities to establish the evaluation system and evaluation index for the characteristics of private schools in China. 
Intelligent teacher evaluation system is the result of objective, fair and practical significance, but also practical strong. The results of SVM method are objective and stable. Combined with the fuzzy comprehensive evaluation method, the complex problem of the teacher evaluation is solved well. Experiments show that this method has good application value and popularization.

\section{Acknowledgments}

This work was financially supported by the Science and Technology Research Project of the Education Department of Jilin Province (2014-B057), the Jilin Province Education Science "Twelve-Five" planning issues (ZD15173), and the Jilin Province Institute higher education research issues (JGJX2015D304).

\section{References}

[1] Donald Christie, The Standard for Chartered Teacher in Scotland, Studies in Educational Evaluation,2006,32:53-72.

[2] Dominique M.A. Sluijsmans, Saskia Brand-Gruwel,Jeroen J.G. van Merrignboer, and Theo J. Bastiaens,THE TRAINING OF PEER ASSESSMENT SKILLS TO PROMOTE THE DEVELOPMENT OF REFLECTION SKILLS IN TEACHER EDUCATION,Studies in Educational Evaluation, 2003,29: 23-42.

[3] LI Honglian,WANG Chunhua,YUAN Baozong, An Improved Support Vector Machines: NNSVM,Chinese Journal of Electronic, 2004,13(2):72-80. [19] Li Changkui,Jiang Chengyu,Wang Ningshen,A Fast Algorithm for Support Vector Clustering, Journal of Southwest Jiaotong University, 2004,12(2).

[4] SUN Jin-wen,YANG Jian-wu,LU Bin,XIAO Jian-guo,Incremental Training for SVM-Based Classification with Keyword Adjusting,Wuhan University Journal of Natural Sciences, 2004(19):5. 